\title{
Métodos unisensoriales para la rehabilitación de la persona con implante coclear y métodos musicoterapéuticos como nueva herramienta de intervención
}

\author{
Unisensorial methods for the rehabilitation of the person with hearing loss \\ and music therapy intervention as a new tool
}

\author{
Yina Quique B', FA MT².
}

\begin{abstract}
RESUMEN
El objetivo de la presente revisión teórica es documentar los aspectos relevantes de los métodos unisensoriales, para la rehabilitación de personas con pérdida auditiva usuarias de implante coclear y otras ayudas auditivas; estos métodos son la terapia auditiva verbal y el método verbotonal, también se incluyen los aspectos encontrados con respecto a la terapia auditiva-oral, la cual es confusa en su definición. Por otro lado se ofrece un panorama de lo que se está realizando desde musicoterapia en esta misma población con el fin de promover puentes de comunicación y trabajo entre fonoaudiología y musicoterapia. Se realizó una búsqueda exhaustiva de información al respecto en bases de datos, decantando y recopilando las referencias obtenidas. Cabe aclarar que el fin de esta investigación no es mostrar la superioridad de ninguno de los tratamientos, sino la forma en la que trabajan para así brindar nuevas perspectivas y herramientas en la intervención con la población usuaria de implantes cocleares u otras ayudas auditivas.

Palabras claves: Deficiencia auditiva, métodos unisensoriales, musicoterapia, método verbotonal, terapia auditiva verbal, fonoaudiología, implante coclear.
\end{abstract}

\begin{abstract}
The aim of this theoretical review is to document the relevant aspects of unisensorial methods in the rehabilitation of people with hearing loss, some of them with cochlear implant or other hearing devices. The methods documented here are Auditory Verbal Therapy and the Verbotonal Method, it is also included some aspects related with the Auditory-Oral therapy which is confused in its definition. The review provides, as well, an overview of what is being done through Music Therapy in this population, in order to
\end{abstract}

1 Universidad Manuela Beltrán, Vicerrectoría de Investigaciones. Colombia.

2 Fonoaudióloga grado de honor Universidad Nacional de Colombia. Magíster en Musicoterapia Universidad Nacional de Colombia. 
promote bridges of communication and work between speech therapy and music therapy. An exhaustive search of databases was conducted decanting and compiling the information obtained. It should be noted that the purpose of this research is not to show the superiority of either approaching, but to show how they work and to bring new perspectives and tools for use in the intervention process with the population using cochlear implants or other hearing devices.

Key words: Auditory Verbal Therapy, Hearing Impairment, Music Therapy, Speech and language pathologist, Unisensorial Methods, Verbotonal Method, cochlear implant.

\section{INTRODUCCIÓN}

La presente revisión teórica pretende ofrecer dos bloques de información, primero un panorama de los métodos unisensoriales para la rehabilitación de la persona con implante coclear (IC), es decir lo referente a la terapia auditiva-verbal y al método verbotonal, también se reportarán aspectos de la terapia auditiva oral. En segundo lugar, se ofrecerá un acercamiento a la musicoterapia, desde sus métodos propios, para esta misma población.

Adicional al objetivo teórico, se quiere destacar la oportunidad de mejora del quehacer terapéutico, a partir de la unión de algunos elementos propios de la fonoaudiología y la musicoterapia, tal sistematización debería redundar en el beneficio de las personas usuarias del IC.

Como fundamento contextual de la revisión teórica, es menester ofrecer una explicación básica del implante coclear, el cual es un dispositivo que recoge el sonido y lo transforma en una señal eléctrica que puede ser interpretada por el cerebro como una señal sonora; el implante se compone de dos piezas, una interna que es colocada en la coclea mediante un proceso quirúrgico y una externa que es visible en la cabeza de los usuarios'.

A nivel internacional se reconoce la importancia de la rehabilitación en los niños que han sido implantados, el informe realizado por la Comisión Española de AudioFonología ${ }^{2}$ sostiene que la mayor parte de los programas encargados de la habilitación-rehabilitación funcionan con cinco etapas de desarrollo auditivo, éstas son: detección, discriminación, identificación, reconocimiento y comprensión.

- La detección hace referencia a la capacidad de indicar la presencia-ausencia de un sonido, se opta primero por incluir en las terapias los sonidos más familiares para el usuario; se progresa realizando reducciones paulatinas en la intensidad; posteriormente, se reduce la duración del estímulo, y por último se realizan variaciones en las frecuencias.

- La discriminación está relacionada con la capacidad del ser humano para catalogar los estímulos sonoros como iguales o diferentes, dentro de la terapia el avance estará marcado por la evolución en la diferenciación de estímulos radicalmente opuestos, hasta llegar a los pares mínimos.

- La identificación hace referencia al reconocimiento auditivo de un estímulo sonoro, dentro de una serie cerrada de alternativas.

- El reconocimiento habla de identificar un ítem verbal con la ayuda de una clave contextual, por ejemplo repetir la frase que oye 0 contestar ante una pregunta 0 una orden, el conjunto de estímulos se presenta en formato abierto.

- Por último, en lo que respecta a la etapa de la comprensión, que es la habilidad que permite la construcción del significado de las palabras y la decodificación de mensajes, la persona debe estar en la capacidad de reconocer un ítem verbal $\sin$ ayuda y de participar en una conversación ${ }^{3}$.

El equipo multidisciplinario encargado del proceso terapéutico, está compuesto generalmente por profesionales en otorrinolaringología, audiología, psicología, terapia ocupacional y fonoaudiología; el fonoaudiólogo conoce las consecuencias que puede dejar la sordera en el desarrollo del lenguaje y la necesidad de realizar tratamientos con enfoque global $^{4}$, por esta razón, ha utilizado herramientas como los métodos unisensoriales, dentro de los que se agrupan la terapia auditiva verbal y el método verbotonal. El campo de acción de la fonoaudiología puede enriquecerse desde la música, con la premisa 
de que los sonidos instrumentales son también una herramienta para el trabajo en las cinco etapas del desarrollo auditivo antes mencionadas, los sonidos son la materia prima del trabajo con usuarios con implante coclear y también son la materia prima de la música.

Utilizar música para rehabilitación no es algo enteramente novedoso, se han encontrado indicios de desarrollos musicales en cada cultura del mundo, incluso las agrupaciones más antiguas muestran patrones sugerentes del uso de los elementos musicales como una herramienta de adoración, recreación y de sanidad. La diferencia es que, en las últimas décadas, se ha asistido al devenir científico que ha aportado conocimiento empírico de las propiedades de la música en las diferentes áreas médicas y en el crecimiento social; la disciplina producto de este devenir se denomina musicoterapia y mediante ésta se han expandido los horizontes en el tratamiento de patologías y se ha mostrado una vez más el valor de la música para la humanidad.

La música ha sido descrita por sus profesionales como un "lenguaje universal", Richard Wagner, Gioacchino Rossini y Christopher North concuerdan con esta afirmación ${ }^{5}$, por lo tanto, puede ser entendida como un vehículo de la comunicación; por su parte, los profesionales en fonoaudiología se especializan en temas relacionados con el lenguaje, el habla y la audición, como elementos esenciales de la comunicación; llama la atención el hecho de que tanto la música como la fonoaudiología se encuentren en el escenario de la comunicación, el abordaje de esta revisión teórica ha sostenido, en el caso de personas con deficiencias auditivas, que la diada fonoaudiología-música vela de forma armoniosa por la rehabilitación de la comunicación.

A pesar de la importancia de la diada mencionada, actualmente se experimenta a nivel mundial una acentuada escasez de conocimiento práctico en el área de música-fonoaudiología en la rehabilitación de personas usuarias de implante coclear, se cuenta con las experiencias mencionadas por las casas de fabricación de implantes cocleares y con algunos documentos en publicaciones periódicas.

Si bien la información disponible es reducida, la población que se beneficiaría, del actuar conjunto de la musicoterapia y la fonoaudiología, es amplia; actualmente en Colombia se estima que existe una población cercana a los 48.202.617 de habitantes, en el año 2004 se identificaron por el DANE (Departamento Administrativo Nacional de Estadística) ${ }^{*}$ un total de 8.977 estudiantes con sordera profunda e hipoacusia en los centros educativos de Colombia 6 . Según el registro para la localización y caracterización de personas con discapacidad $^{7}$, se reportan 99.693 personas, residentes en Colombia, con dificultades permanentes para oír, algunos datos relevantes de caracterización de esta población son:

"- La prevalencia de personas con dificultad para oír, es reportada en mayor proporción en mujeres que en hombres $(50,2 \%$ son mujeres y $49,8 \%$ son hombres).

- La mayoría de los estudiantes con dificultad auditiva se encuentra en educación básica primaria.

- La mayoría se encuentra afiliada al Régimen de Seguridad Social en Salud (74\%), y la principal vinculación es al régimen subsidiado.

- El $43 \%$ del total registrado vive en estrato $1^{* *}$.

- El $69,5 \%$ del total registrado vive en áreas urbanas".

Hasta este punto se ha presentado información desde la perspectiva colombiana, sin embargo, desde una óptica internacional se tiene que la OMS (Organización Mundial de la Salud) reveló, en 2005, una cifra de 278 millones de personas con defectos de audición moderados o profundos 8 , es decir que existe un vasto potencial de usuarios de implante coclear a nivel mundial y por lo tanto una oportunidad manifiesta, para los profesionales de la fonoaudiología, de ofrecer nuevas alternativas de rehabilitación, en este caso, mediante la combinación de la música con los métodos que han conocido de antemano.

\footnotetext{
* Entidad responsable de la planeación, levantamiento, procesamiento, análisis y difusión de las estadísticas oficiales de Colombia.

** La estratificación en Colombia es una clasificación que tiene en cuenta diversos factores económicos y sociales para determinar los rangos de valor de impuestos y tarifas de servicios públicos. La estratificación puede ser un indicador de la clase social a la que pertenecen los ciudadanos así: de 0 a 2 clase baja; de 3 a 5 clase media y 6 clase alta.
} 


\section{MATERIALES Y MÉTODOS}

La búsqueda de información y el cuerpo de la presente revisión teórica se dividió en dos áreas principales: la primera de ellas abarca los métodos orales unisensoriales para la rehabilitación de la persona con implante coclear; la segunda área comprende la presentación de los métodos musicoterapéuticos como nueva herramienta de rehabilitación para pacientes con implante coclear.

Como fuentes de información se acudió a bases de datos como Pubmed y Ebsco mediante la búsqueda de palabras como: Verbotonal, Auditory Oral Therapy, Auditory Verbal Therapy, Music Therapy, Cochlear Implant y Music Therapy; vale aclarar que el objetivo de esta revisión teórica no es mostrar la superioridad de ninguna de las alternativas de intervención, sino la exposición de las diversas formas en las que trabajan, con el fin de brindar nuevas perspectivas y herramientas en el trabajo con la población usuaria de implantes cocleares.

\section{Revisión teórica}

El primer abordaje desde el que se tratan, en la literatura, los problemas auditivos es la perspectiva profiláctica, es decir, el despliegue de los recursos pertinentes que tiendan a evitar los efectos de los problemas auditivos; desde tal enfoque se valora el hecho de que la identificación y diagnóstico temprano de problemas en la audición puedan conducir a la adaptación de auxiliares auditivos y, consecuentemente, al desarrollo de programas de rehabilitación ${ }^{9}$.

Para conocer el concepto de prevención y rehabilitación en Colombia se recurrió a la Ley 324 de 1996, mediante la cual se formularon algunas normas a favor de la población sorda; la ley define la prevención como aquellas medidas que se encaminan a impedir que se produzca un deterioro físico, intelectual, psiquiátrico o sensorial (Prevención primaria) por una parte; 0 a impedir que ese deterioro cause una discapacidad o limitación funcional permanente (Prevención secundaria) por otra parte. A su vez la rehabilitación está definida como un proceso orientado a lograr que las personas con discapacidad estén en condiciones de alcanzar y mantener un estado funcional óptimo desde el punto de vista físico, sensorial, intelectual, psíquico o social, de manera que cuenten con medios para modificar su propia vida y alcanzar, paulatinamente, una mayor independencia ${ }^{10}$.

Desde un análisis fonoaudiológico la producción de la palabra y del lenguaje están relacionadas con el aprendizaje auditivo ${ }^{11}$. La fonoaudiología plantea tres etapas en la rehabilitación: la primera se ocupa de los aprendizajes básicos; sus dos propósitos son asentar las bases del lenguaje e impulsar los procesos cognitivos. La segunda está encargada de la consolidación del lenguaje, lo cual coincide con el ingreso al ámbito escolar; en esta etapa se requiere un acompañamiento constante para la conciliación y el éxito escolar. La tercera se ocupa del aprendizaje lecto-escrito.

Antes de enfocar el tratamiento desde un abordaje específico, sea este enfoque monolingüista, bilingüista 0 su mixtura, se puede optar por una ayuda protésica (ver Figura 1) ${ }^{12}$. Si se decide por el primer abordaje (monolingüismo u oralismo) los intervinientes en el proceso de rehabilitación realizan todos los esfuerzos para conseguir la máxima competencia oral en la lengua materna de la persona con deficiencia auditiva; en la segunda opción (bilingüismo sea con Lengua de Señas Colombiana - LSC o con español signado) los intervinientes consideran la habilidad de la persona con deficiencia auditiva, para comunicar en lengua viso-gestual y para adquirir, por medio de este lenguaje, el conocimiento ${ }^{13}$. En otras palabras, desde una perspectiva oralista se plantea la adquisición del lenguaje hablado, poniendo énfasis en el entrenamiento auditivo para desarrollar la percepción, producción y comprensión del habla; desde una perspectiva gestualista se propone restablecer, lo antes posible, la interacción comunicativa por medio de la adquisición de un sistema lingüístico alternativo ${ }^{14}$.

Escoger el método de tratamiento ha sido motivo de controversia a lo largo del tiempo ${ }^{15}$, en esta elección los padres juegan un papel fundamental en el que adoptan la mejor opción comunicativa y educacional ${ }^{16}$ para sus hijos, teniendo en cuenta que se debe empezar a intervenir lo más pronto posible para lograr un desarrollo cognitivo y lingüístico que permita el éxito escolar ${ }^{14}$. 


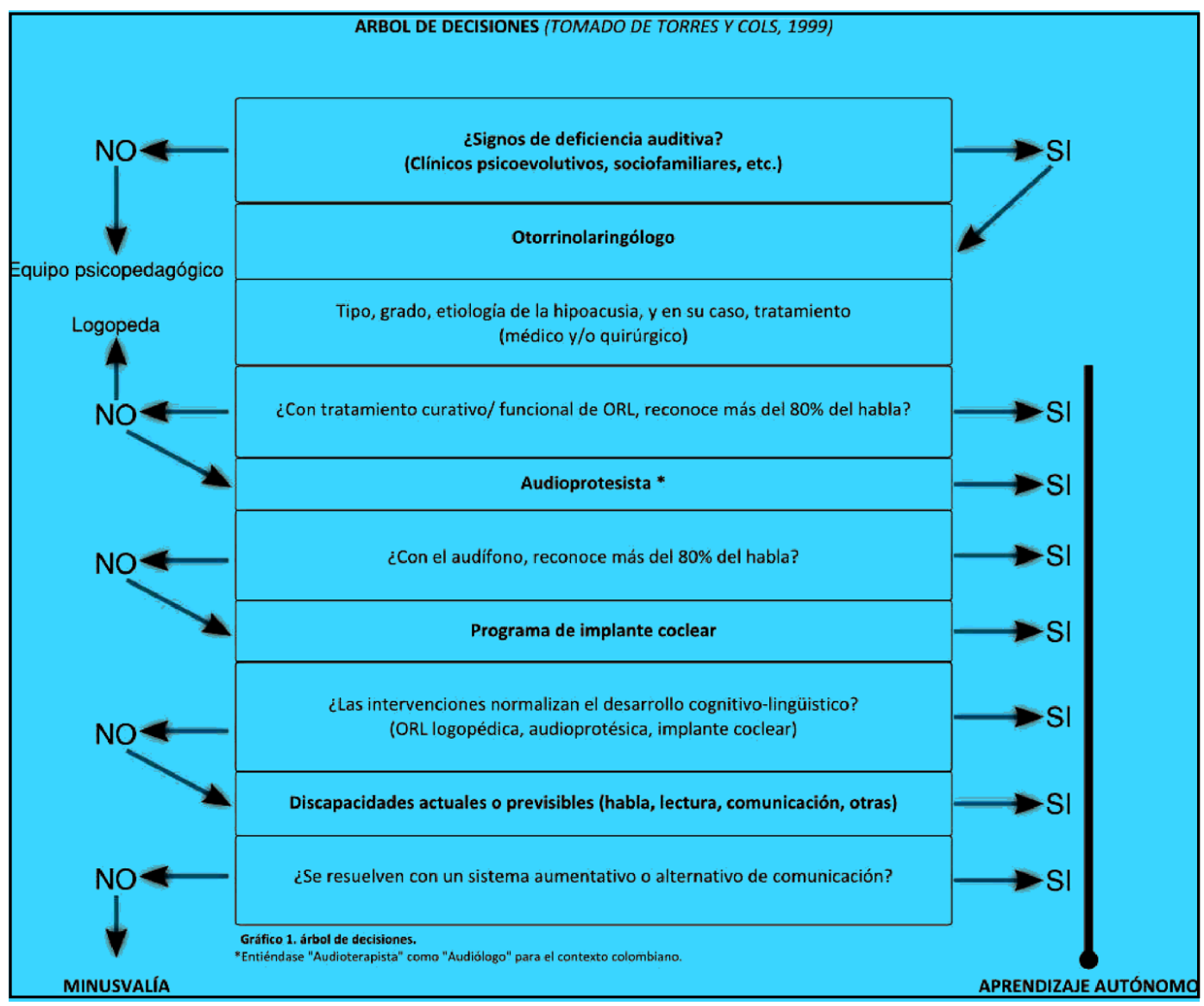

Figura 1.

\section{Terapia auditiva-verbal (TAV)}

La TAV se podría definir brevemente como la aplicación de tecnología, sumada a estrategias, técnicas y procedimientos para habilitar la escucha y el entendimiento del lenguaje, en los niños con dificultades auditivas con el fin de que puedan comunicarse a través del habla usando su ayuda auditiva $^{17}$

Tuvo sus orígenes en el método acústico de Urbantschitsch de Viena, a principios del siglo XX; hacia los años 20 fue llevado a los Estados Unidos por el Dr. Goldstein, allí el método fue tomando diferentes nombres de acuerdo a los grupos que lo utilizaron: en Holanda el Dr. Huizing y en Estados Unidos la Dra. Pollack lo denominaron Enfoque Acupédico, en Londres el Dr. Whetnall lo nombró "Enfoque Auditivo", en Suecia el Dr. Wedenberg usó las palabras "Enfoque Unisensorial", el Dr. Tsunoda en Japón lo describió como programa de entrenamiento auditivo unisensorial, Agnes y Ling desarrollaron el método en Canadá. Hoy en día, la organización Auditory Verbal International AGBell, ubicada en los Estados Unidos agrupa a los profesionales y familias comprometidas con la difusión de la Terapia Auditivo Verbal' ${ }^{18}$.

\section{Principios de la TAV'8}

- Realizar una detección e identificación temprana de las dificultades auditivas en niños.

- Apropiada amplificación y/o tecnología de implante coclear con el fin de alcanzar el máximo aprendizaje a través de la escucha.

- Realización constante de evaluaciones audiológicas, ningún test es considerado redundante. 
- Búsqueda, durante el proceso de rehabilitación, de ambientes auditivos que favorezcan la adquisición del lenguaje hablado, incluyendo la terapia individualizada.

- Integrar la escucha a todo el "ser" del niño, es decir, que escuchar se convierta en un estilo de vida.

- Constante proceso de evaluación de la evolución del desarrollo auditivo, habla, lenguaje y cognición en cada encuentro de trabajo

- Integración e incorporación de niños con pérdida auditiva a la educación regular.

- Participación activa de padres para mejorar la comunicación hablada entre el niño y los miembros de la familia.

- Afirmación de los padres como modelos primarios para que los niños aprendan a escuchar su propia voz, las voces de otros, los sonidos del ambiente y el lenguaje hablado.

- Integración de habla, lenguaje, audición y cognición en respuesta a las necesidades psicológicas, sociales y educativas del niño y la familia.

\section{Estrategias de la TAV}

- Realce acústico: Son estrategias para mejorar la audición, el terapeuta se mueve desde lo más audible hacia lo menos audible incluyendo herramientas como el susurro, el canto y el énfasis de los aspectos segmentales-suprasegmentales.

- Acercamiento auditivo: Hace referencia a la ubicación del terapeuta cerca del mejor oído, en términos auditivos.

- Parafraseo: Consiste en dar la misma consigna usando diferentes recursos oratorios para su comprensión.

- Preguntar: Es recibir una retroalimentación constante del niño por medio de la pregunta_qué escuchaste?

- Pausas: Se refiere a darle tiempo al niño para que dé una respuesta.

- Expansión: Hace referencia a la comunicación de una mayor cantidad de características de un determinado elemento.

- Sandwich auditivo: Es una estrategia que comienza por hacer que el niño escuche sin apoyo visual, luego con apoyo visual del objeto y por último se utiliza únicamente el estímulo auditivo.
- Condicionamiento auditivo: Se relaciona con la habilidad de determinar la presencia 0 ausencia del sonido, cada vez que el niño escucha un sonido debe generar una respuesta.

\section{Participación de padres}

Generalmente los padres tienen expectativas altas frente a los resultados de la TAV ${ }^{19}$, en las sesiones de trabajo ellos necesitan observar y practicar técnicas para estimular habla, lenguaje y cognición, además planear estrategias para integrarlas en las rutinas y experiencias diarias. También deben informar al terapeuta acerca de los intereses y habilidades del niño, desarrollar técnicas de manejo de comportamiento adecuado, grabar y discutir partes del proceso, entender los objetivos a corto y a largo plazo, desarrollar la confianza en la interacción de padres-hijos y tomar decisiones en favor de sus hijos. En resumen, la práctica auditiva verbal apoya la participación de los padres buscando que los niños con pérdida auditiva crezcan en un aprendizaje y ambiente regular para que adquieran independencia y participación en la sociedad ${ }^{20}$.

\section{Reportes de la literatura acerca de la TAV}

Un estudio longitudinal, cuyo seguimiento tuvo una duración de más de 50 meses, comparó un grupo experimental de 29 niños pertenecientes a programas de TAV con pérdida auditiva severa (promedio tonos puros - PTA de 79,37 dB HL), con un grupo control de niños oyentes; los grupos fueron empalmados por edad de lenguaje, vocabulario receptivo, género y ubicación socioeconómica. Los resultados mostraron avances positivos en el habla y el lenguaje del grupo experimental y concluyeron que la TAV es efectiva para la población de niños con pérdida auditiva ${ }^{21}$.

Otro estudio examinó la percepción del habla, el habla y el desarrollo del lenguaje en 25 niños, pertenecientes a un programa de TAV, con hipoacusia severa (promedio de tonos puros - PTA $79,37 \mathrm{~dB} \mathrm{HL}$ ); se realizaron evaluaciones separadas por un periodo de 21 meses. Los resultados mostraron que los niños mejoraron su percepción del habla a lo largo del tiempo y su rango de progreso en habla y lenguaje fue similar al de niños con audición típica22.

Al evaluar el habla, el lenguaje y la percepción del habla en ruido, antes y después de 20 semanas 
de tratamiento con TAV, en una población de 7 niños con pérdida neurosensorial profunda, se observan avances significativos en la percepción y producción del habla ${ }^{23}$.

En un estudio realizado en 65 padres de hijos que participaban de programas de TAV, se reporta que existen efectos positivos a largo plazo de este tratamiento, también se reitera la importancia de los padres como miembros inalienables del proceso de rehabilitación ${ }^{24}$.

En un trabajo prospectivo, realizado en 47 niños sordos prelinguales, se midió la inteligibilidad del habla espontánea de cada niño antes y en intervalos de 5 años después de la implantación coclear, todos participaron de TAV luego de la activación de su implante, los resultados muestran que el rango de inteligibilidad incrementó significativamente cada año después de la rehabilitación ${ }^{25}$.

En otra mirada prospectiva, realizada sobre 42 adultos usuarios de implante coclear, se incluyeron la terapia auditiva verbal temprana, el apoyo familiar y una actitud positiva como los factores más comunes citados en el éxito del uso del implante ${ }^{26}$. En otro estudio los resultados muestran que el $57 \%$ de los 72 usuarios con pérdida auditiva de moderada a profunda, que permanecieron en TAV durante más de un año, pudieron integrarse completamente a la educación regular, por lo cual se concluye que este tipo de terapia ofrece una exitosa intervención a los estudiantes ${ }^{27}$.

En un estudio realizado en 10 usuarios de IC con pérdida auditiva secundaria a meningitis, la TAV, junto a otros factores, se identificó como un elemento para preservar el control a largo plazo de la frecuencia y amplitud de las pérdidas auditivas neurosensoriales secundarias a meningitis ${ }^{28}$.

La investigación también ha profundizado la influencia del género en el desarrollo del lenguaje en el marco de la TAV, en este estudio realizado a los padres de 28 niños y 42 niñas se consideró el comportamiento de los menores asistentes a un programa clínico de TAV; Ios resultados reportan que las niñas superaron a los niños aunque ambos grupos tuvieron resultados positivos ${ }^{29}$.

En lo referente a los estándares de la práctica basada en la evidencia, que apoya la TAV como una aproximación de intervención, se categorizó la información en términos de fuerza científica y se concluyó que existen algunos tipos de evidencia que aún necesitan apoyo, por lo cual se anima a los agentes generadores de conocimiento a la colaboración y generación de evidencia ${ }^{30}$.

Se concluye que la terapia auditiva verbal es un método de rehabilitación para personas sordas usuarias de ayudas auditivas como el implante coclear, dentro de su filosofía involucra de forma activa a los padres con el fin de aprovechar los espacios que se comparten en familia en pro del desarrollo de habilidades comunicativas y auditivas. La capacitación en TAV es costosa y requiere una significativa inversión de tiempo, tal impedimento, aunado a las barreras económicas y sociales, son los retos con los que lidian profesionales y padres, en el contexto colombiano, en contraste con países, de otras latitudes, en donde los padres reciben un subsidio para compartir más tiempo con sus hijos y tener mayores oportunidades de éxito con la TAV.

\section{Método verbotonal}

En un comienzo las bases científicas del Sistema Verbotonal se sustentaron en las investigaciones fonéticas cuyo punto de partida fue la percepción y comprensión auditiva; un segundo momento dio paso a los trabajos verbotonales que pueden entenderse como una ampliación de las investigaciones en el campo de la fonética experimental, estos trabajos ofrecen evidencia de que cada sonido o palabra tiene su campo frecuencial óptimo, el cual abarca una octava, que no necesariamente corresponde con sus frecuencias formantes; fuera de su octava óptima el sonido 0 palabra es percibido como otro distinto.

Los especialistas en el método verbotonal indican que intentar resumirlo entraña cierta dificultad por su metodología y técnica, por lo cual, generalmente afirman que es una forma de intervención que pretende ser globalizada y exhaustiva en todos los niveles de estructuración del habla y del lenguaje ${ }^{31}$. En pocas palabras se podría afirmar que el método verbotonal es la aplicación del sistema verbotonal al ámbito de la sordera ${ }^{32}$, tal aplicación considera todos los "actos del lenguaje", no sólo como producciones orales, sino que también estima como elementos de soporte comunicativo al cuerpo, al ritmo y entonación, a la expresividad y afectividad, y al contexto. 
Este método toma elementos de la percepción auditiva y táctil en donde la palabra hablada se usa como estímulo ${ }^{33}$, se ejecuta por medio de aparatos SUVAG (Sistema Universal Verbal Auditivo de Guberina), Requerimientos pedaglos cuales son amplificadores de sonidos con vibradores, micrófonos y cascos. Un principio básico de la investigación y rehabilitación verbotonal es que todo el cuerpo humano funciona como receptor y transmisor, las investigaciones llevadas a cabo en uno de Ios centros SUVAG* han revelado que el cuerpo humano vibra con frecuencias entre 7 y $12 \mathrm{~Hz}$, por esto el procedimiento básico inicial en la rehabilitación de la persona con déficit auditivo se apoya en la vibración ${ }^{34}$.

Guberina, su principal exponente, plantea que las personas con pérdida auditiva tienen zonas frecuenciales en las cuales son más sensibles, es decir donde se conserva la posibilidad de escucha, por esto utiliza el campo óptimo de audición y evita el reclutamiento ${ }^{35}$.

Para elaborar un programa de trabajo es necesario realizar una evaluación que refleje las capacidades y el estado actual de la persona en tres áreas concretas: área auditiva, en la cual se analizan las capacidades en el campo óptimo, se lleva a cabo con audífonos y sin ninguna amplificación; área fonética/fonológica, en la que se exploran aspectos como la voz, los elementos prosódicos, la intensidad y la articulación; por último, en el área lingüística se explora la comprensión y expresión del lenguaje del niño $0^{34}$.

\section{Principios generales de la rehabilitación verbotona $^{34}$}

- Orientación espacial: Mejorar la orientación en el espacio mejora la función auditiva, ampliando las posibilidades de percibir los estímulos sonoros del medio ambiente y del material léxico.

- Campo óptimo de la audición: Cada persona con dificultad en la audición presenta restos auditivos que son identificados en el diagnóstico verbotonal, éstos se aíslan para utilizar los estímulos necesarios en el proceso de aprendizaje.
- Ampliación funcional del campo óptimo de la audición: En cada sesión de entrenamiento se busca ampliar el campo óptimo de la audición, la progresión empieza con la detección del sonido, continúa con la discriminación e identificación y termina en la comprensión sonora.

- Control auditivo: Se busca regular la pronunciación, aumentar la comprensión mediante la lectura labiofacial y ofrecer progresivamente mejores bases para la comunicación.

- Seguir la progresión lingüística del niño oyente: La intervención con vibración o ayudas auditivas permiten que los componentes del sonido formen estructuras rítmicas bases para las estructuras lingüísticas. Se aconseja hablarle al niño constantemente y participar del tratamiento individual, de los ritmos fonéticos (corporal y musical) y de la clase verbotonal.

\section{Reportes de la literatura acerca del método verbotonal}

Como se ha mencionado, el método verbotonal se basa en el modelo de desarrollo de personas con audición normal y enfatiza la importancia del ritmo, entonación y cualidad vocal en personas con deficiencias auditivas. El personal clínico entrenado en este método utiliza amplificación de alta calidad y las entradas vibrotáctiles de Ios SUVAG para el tratamiento; se ha evidenciado una integración, a la educación regular, de entre $60 \%$ y $90 \%$ de los asistentes a este método terapéutico ${ }^{36}$ y una exitosa aplicación clínica ${ }^{37}$.

En un estudio que comparó la percepción del habla de 29 niños con implante coclear con 20 usuarios de otras ayudas auditivas, ambos grupos con pérdidas auditivas profundas, se evaluaron tareas de diferenciación de pares mínimos encontrándose diferencias significativas positivas en niños con implante coclear que asistían a un programa de método verbotonal ${ }^{38}$.

Los resultados de un estudio que reunió los efectos de un proceso de tratamiento de 5 años con el método verbotonal, en donde participaron 80

\footnotetext{
* Los centros SUVAG son instituciones establecidas en diferentes lugares del mundo, dispuestas para la rehabilitación de la escucha y el habla de personas con deficiencias auditivas que han optado por el método verbotonal.
} 
adultos con pérdidas auditivas de moderadas a profundas, revelaron que el $71 \%$ de los pacientes mostró un avance significativo en la percepción auditiva, se resalta que este método es útil ya que identifica funciones auditivas que no son frecuentemente detectadas en procedimientos audiométricos. Se agrega que como un método de rehabilitación aural es particularmente benéfico en personas que alcanzan buenos puntajes en los test de discriminación pero no funcionan bien en las situaciones de escucha cotidiana o también en los casos en los que las personas no puedan ajustarse a la amplificación $^{39}$.

En un estudio, que incluyó 50 estudiantes sordos de una guardería, los resultados concluyeron que el habla de los niños enseñados con el método verbotonal mejoró de forma significativa en comparación con las aproximaciones convencionales ${ }^{40}$.

Se resume, a partir de los resultados descritos, que el método verbotonal es utilizado en los procesos de rehabilitación de la persona con deficiencia auditiva, incluyendo usuarios de implante coclear, sin embargo, en el contexto colombiano no se cuenta con acceso suficiente a los equipos SUVAG, por lo cual su práctica no está muy difundida, además, durante la búsqueda de información, no se cosechó un abundante número de publicaciones al respecto, por lo cual no fue posible caracterizar su práctica reciente en otros países.

\section{¿El método auditivo-oral?}

A continuación se comentarán algunos reportes de lo que se ha denominado el método auditivo-oral, se aclara que, pese a apoyarse en diversas consultas con profesionales del área de la fonoaudiología, no ha sido posible llegar a una definición nítida de este método, su uso o sus principales exponentes; pareciera ser un problema de denominación en el que en la literatura se ha usado el nombre auditivooral indistintamente, en asociación con la TAV o con el método verbotonal, de allí que en ciertos momentos aparenta una estrecha relación con el método verbotonal y en otras con el método auditivo- verbal, en cualquier caso sin una explicación clara de lo que diferencia el método auditivo-oral de los demás. Teniendo en cuenta la anterior aclaración los siguientes párrafos se limitarán a resumir los hallazgos patentes en la literatura.

En un estudio cruzado que evaluó la inteligibilidad de un grupo de 57 adolescentes usuarios de implante coclear, en programas auditivo orales versus comunicación total ${ }^{*}$, se encontraron altos niveles de precisión en ambos grupos ${ }^{41}$. Se concluyó que la gran mayoría de los usuarios con implante coclear realizan mejoras significativas en la percepción del habla, sin embargo se reconoce una falla en ambas filosofías (auditiva-oral y comunicación total), puesto que, generalmente, la marca de "la voz del sordo" es una característica de ambos programas ${ }^{42}$.

Otro estudio comparó el control conversacional de madres oyentes, quienes usaban comunicación aural-oral, en comparación con las que usaban comunicación total; los resultados indicaron que las madres en ambos grupos ejercieron niveles altos de control conversacional, tales niveles resultaron similares entre ambos grupos; en el grupo de comunicación aural-oral el estilo maternal reflejó la habilidad del niño en el lenguaje hablado; en el grupo de comunicación total el estilo maternal se relacionó con el desarrollo social del niño $0^{43}$.

En un estudio realizado en 18 sujetos sordos prelinguales, usuarios de implante coclear, se menciona que la presencia de una entrada auditiva, por medio de las ayudas auditivas, antes de la implantación coclear, sumada a la terapia auditiva-oraly a un buen nivel de educación, puede influenciar positivamente los resultados del implante coclear en sorderas prelinguales ${ }^{44}$.

Los resultados que obtuvieron 22 usuarios con implante coclear, quienes asistieron en lapsos de entre 1 y 7 años, a un programa auditivo-oral intensivo, excedieron los obtenidos previamente, en estudios similares, con sordos profundos, usuarios de otras ayudas auditivas; el $50 \%$ de la población, participante del estudio, obtuvo puntajes que se encuentran en el rango de oyentes normales, el estudio concluyó que los niveles de

\footnotetext{
* La comunicación total podría resumirse como un sistema de comunicación bimodal, que utiliza simultáneamente el código oral y el signado.
} 
desarrollo del lenguaje y de lectura están asociados con implantación temprana y un ambiente abierto para la percepción del habla ${ }^{45}$.

La gran mayoría de los niños usuarios de implante coclear desarrollan una menor cantidad de vocabulario que sus pares oyentes, sin embargo, un estudio realizado en 65 niños sordos, usuarios de IC, educados en un ambiente auditivo oral, evidenció un incremento importante de vocabulario en los participantes; este estudio concluyó que la curva de crecimiento, de quienes han sido implantados cerca a los dos años de edad, puede alcanzar cotas similares al promedio de los niños oyentes ${ }^{46}$. En el mismo sentido un estudio análogo, llevado a cabo con 27 niños con sordera profunda, concluyó que una aproximación auditivo-oral extendió la longitud del habla y el vocabulario de sus participantes ${ }^{47}$.

A manera de conclusión, respecto del método auditivo-oral, se reporta que la literatura contiene estudios que utilizan el método auditivo-oral para la rehabilitación de personas con deficiencia auditiva, incluyendo usuarios de implante coclear, sin embargo al profundizar en la búsqueda de orígenes y exponentes no se encuentra una definición clara o una asociación con alguno de los métodos descritos anteriormente, por lo cual, este método continúa planteando una incógnita que debería encontrar solución desde la investigación en el quehacer fonoaudiológico.

\section{MUSICOTERAPIA}

La musicoterapia es el uso de la música y/o de los elementos musicales, por un musicoterapeuta calificado, con un paciente o grupo de pacientes; su objetivo es facilitar y promover la comunicación, la interrelación, el aprendizaje, la movilización, la expresión, la organización y el alcance de otras metas terapéuticas relevantes, con el objeto de atender necesidades físicas, emocionales, mentales, sociales y cognitivas ${ }^{48}$.

\section{Métodos en musicoterapia ${ }^{49}$}

- Improvisación: En este tipo de experiencias el paciente crea música al cantar o tocar un instrumento (incluyendo la voz), mediante la proposición espontánea de la melodía, el ritmo, la canción o la pieza instrumental. Las improvisaciones pueden realizarse en compañía del terapeuta, del grupo o individualmente.

- Recreativo: En estas experiencias el usuario aprende 0 ejecuta música vocal 0 instrumental, que puede haber sido compuesta previamente, o reproduce cualquier forma musical que se haya presentado como modelo, dicha ejecución puede realizarse ante una audiencia aunque no es un requisito imperativo.

- Composición: En este tipo de experiencias el terapeuta ayuda al paciente a escribir canciones, letras o piezas instrumentales, 0 a crear algún tipo de producto musical como material audiovisual o grabaciones en audio. Normalmente el terapeuta es responsable de los aspectos técnicos del proceso y diseña la participación del usuario según sus habilidades musicales.

- Receptivo: Aquí se encuadran las experiencias en donde el paciente escucha música y responde a la experiencia de forma silenciosa, verbal, cinética, artística u otras diversas que se rigen por la espontaneidad del paciente. La experiencia de escucha puede focalizarse en los aspectos físicos, emocionales, intelectuales, estéticos 0 espirituales de la música.

\section{Musicoterapia e implante coclear}

Las autoras de un estudio, que dio cuenta del estado del arte de musicoterapia en IC incluyó un total de 11 publicaciones a nivel mundial, encontraron que la mayor cantidad de información, en dicha materia, se encuentra en revistas médicas, en trabajos presentados en congresos y en conferencias ${ }^{50}$; de lo anterior se puede concluir la escasez de reportes sobre tratamientos realizados a partir de musicoterapia en usuarios de implante coclear, por Io cual, en este apartado se describirán someramente, algunas guías que pueden regir los procesos y algunas experiencias referidas en la literatura.

Mediante la experiencia musicoterapéutica se ha logrado descubrir que los niños implantados pasan por las mismas etapas de desarrollo de audición por las que pasan los niños con audición normal con la diferencia de que lo hacen unos años más tarde, por eso es importante brindarles apoyo 
y motivación a través de las experiencias cotidianas $^{51}$. Se ha propuesto que la musicoterapia brinda un espacio de rehabilitación y de descubrimiento de la creatividad en los niños con IC; se menciona la improvisación como una herramienta clave para el trabajo con esta población ${ }^{52}$.

Consejos para el programa de musicoterapia en IC ${ }^{53}$

1. Establecer expectativas realistas de escucha.

2. Ayudar a los usuarios a seleccionar ejemplos musicales que sean más fáciles para el IC, por ejemplo empezar escuchando únicamente la letra de las canciones, tener ejemplos con ritmos fuertes y claros, o usar melodías simples, entre otros.

3. Escuchar en ambientes óptimos, es decir con buenos equipos reproductores de sonido, en una habitación con poco eco y sin ruido de fondo. Realizar ensayos cortos pero frecuentes, esto es, con una duración de 10 a 15 minutos 0 aun menos, de ser necesario. Escuchar las mismas selecciones de música muchas veces durante varios días, ello en la medida en que repetir la exposición es un factor importante para mejorar el reconocimiento y mejorar la cualidad del tono.

4. Usar el contexto y otras claves para que el sonido tenga sentido, se les puede animar a seguir claves visuales como mirar los labios de los cantantes o las manos de los instrumentistas para seguir el ritmo, también se puede sugerir seguir las letras de las canciones.

5. No sentir miedo de experimentar, los usuarios que han logrado disfrutar la música no han tenido miedo de tratar con diferentes y nuevos sonidos.

\section{Reportes de la literatura acerca de musicoterapia}

Se ha mencionado, en la literatura, que el primer objetivo de musicoterapia en la población de usuarios con IC, es despertar un interés por los instrumentos musicales y por la exploración del sonido, se manifiesta que es más fácil alcanzar la percepción de la música que la percepción del lenguaje debido a factores como la intensidad y las frecuencias que la caracterizan. La siguiente meta es animarlos tanto a descubrir como a distinguir, identificar y entender los sonidos; en este punto, los niños, encuentran una nueva forma de expresar las emociones ya que el habla no es su medio más eficaz para hacerlo, por esto deben experimentar la música como algo positivo y estimulante, capaz de enriquecer su percepción, la autora recalca que el tema de mayor relevancia es la comunicación no ligada a la comunicación verbal51.

En una revisión teórica consultada, se menciona que algunos fragmentos musicales e incluso ciertos estilos pueden ser percibidos, con mayor efectividad, por medio del IC, la razón de ser de esta circunstancia es que no toda la música comparte los mismos rasgos; generalmente los usuarios de IC prefieren música con beats acentuados, como el rock o rap ${ }^{54}$.

La música y el habla son similares en el sentido en que están constituidas por patrones de sonido que se desarrollan a través del tiempo. Por un lado, el habla requiere una articulación rápida y los aspectos de la señal cambian continuamente, por ejemplo el rango típico de una conversación en inglés, incluyendo las pausas, es de 12.5 sonidos perceptibles por segundo; por su parte la música tiene un rango considerablemente más lento con tonos individuales sostenidos de 200 a 600 milisegundos. Por otro lado el tono, que establece contrastes lingüísticos en el habla (oraciones y preguntas), es relativamente largo, de forma que se acerca al orden de 6 semitonos; en contraste, la música presenta cambios mucho más pequeños que juegan un papel significativo (un semitono hace la diferencia entre un acorde mayor y uno menor) y las melodías con notas sucesivas generalmente están separadas por 1 ó 2 semitonos ${ }^{55}$. Se han hallado correlaciones entre la capacidad de percibir la música y la comprensión del habla en ambientes ruidosos, así el mejoramiento de la percepción musical podría ofrecer, un beneficio médico más amplio en la distinción de la figurafondo ${ }^{56}$.

Aunque muchos adultos que reciben un IC después de haber perdido su audición encuentran la música inaceptable, los niños, usuarios de implantes, que nacieron sordos o adquirieron la sordera cuando eran pequeños encuentran la música interesante y disfrutable; ese segundo tipo de usuarios puede reconocer canciones populares que han sido escuchadas regularmente, también puede identificar el tema de algunas canciones de programas de televisión, e incluso, luego de algu- 
nos meses de haber recibido el implante, los niños prefieren el canto al silencio ${ }^{57}$. En general se apoya la idea de que la experiencia musical puede contribuir en la calidad de vida de personas con audición típica y con problemas de audición ${ }^{58}$.

Otra investigación, realizada a 6 niños con sordera profunda usuarios de IC, reportó que la aplicación de un programa de musicoterapia beneficia la detección, discriminación, identificación y comprensión sonora de niños con IC; además destacó el desarrollo del "ser" y el "hacer" musical evidenciado en cada uno de los niños durante y al final del programa ${ }^{59}$.

Los métodos en musicoterapia son una herramienta novedosa para el tratamiento de personas con implantes cocleares, es necesario seguir investigando con el fin de conocer sus efectos desde el punto de vista de la práctica basada en la evidencia, sin embargo, los reportes de la percepción musical, el mejoramiento de calidad de vida y

Tabla 1. Paralelo entre aspectos de los métodos unisensoriales para la rehabilitación de la persona con implante coclear y los métodos musicoterapéuticos*

\begin{tabular}{|c|c|c|c|}
\hline Aspectos & Terapia auditiva verbal & Método verbotonal & Musicoterapia \\
\hline ¿Qué es? & $\begin{array}{l}\text { Es un enfoque terapéutico } \\
\text { para la rehabilitación de } \\
\text { personas con deficiencias } \\
\text { auditivas que enfatiza en las } \\
\text { habilidades auditivas } \\
\text { para desarrollar el } \\
\text { lenguaje y el habla. }\end{array}$ & $\begin{array}{l}\text { Es la aplicación del } \\
\text { sistema verbotonal en el } \\
\text { ámbito de la deficiencia } \\
\text { auditiva, incluye el } \\
\text { trabajo individual, el ritmo } \\
\text { musical y corporal y la } \\
\text { clase verbotonal. }\end{array}$ & $\begin{array}{l}\text { Es el uso de la música, y de } \\
\text { los elementos musicales, por } \\
\text { un musicoterapeuta calificado, } \\
\text { para promover la comunicación, } \\
\text { es aplicable a usuarios } \\
\text { con pérdida auditiva } \\
\text { incluyendo el implante coclear. }\end{array}$ \\
\hline $\begin{array}{l}\text { ¿Cuáles son } \\
\text { sus orígenes? }\end{array}$ & $\begin{array}{l}\text { Se origina en el método } \\
\text { acústico de Urbantschitsch } \\
\text { de Viena, a principios } \\
\text { del siglo XX. }\end{array}$ & $\begin{array}{l}\text { Sus orígenes se remontan al } \\
\text { año 1954, su creador fue } \\
\text { un lingüista croata llamado } \\
\text { Petar Guberina. }\end{array}$ & $\begin{array}{l}\text { La idea de la música ejerciendo } \\
\text { una influencia curativa es antigua, } \\
\text { los primeros papiros egipcios, } \\
\text { relacionados al tema, se } \\
\text { encontraron en el año } 1889 \text {, sin } \\
\text { embargo el devenir científico de la } \\
\text { musicoterapia surgió en el siglo XX. }\end{array}$ \\
\hline $\begin{array}{l}\text { ¿Requiere } \\
\text { entrenamiento } \\
\text { formal? }\end{array}$ & Sí & Sí & Sí \\
\hline $\begin{array}{l}\text { ¿Existe una } \\
\text { participación } \\
\text { activa de } \\
\text { padres dentro } \\
\text { del programa de } \\
\text { rehabilitación? }\end{array}$ & $\mathrm{Si}$ & Rol secundario & Rol secundario \\
\hline $\begin{array}{l}\text { ¿Aplicado en } \\
\text { instituciones } \\
\text { en el contexto } \\
\text { colombiano? }\end{array}$ & $\begin{array}{l}\text { Se cuentan con las } \\
\text { experiencias de CINDA } \\
\text { Centro de investigación } \\
\text { e información en deficiencias } \\
\text { auditivas y la Clínica } \\
\text { José A. Rivas. }\end{array}$ & $\begin{array}{l}\text { Se cuenta con la experiencia } \\
\text { de la Fundación para el } \\
\text { Niño Sordo - ICAL. }\end{array}$ & $\begin{array}{l}\text { Hasta el momento no se reporta } \\
\text { una institución específica, } \\
\text { sin embargo, es aplicada en } \\
\text { la práctica privada por personas } \\
\text { entrenadas en musicoterapia. }\end{array}$ \\
\hline $\begin{array}{l}\text { ¿Cuáles son } \\
\text { sus principios } \\
0 \text { estrategias } \\
\text { de trabajo? }\end{array}$ & $\begin{array}{l}\text { Realce acústico, acercamiento } \\
\text { auditivo, parafraseo, preguntar, } \\
\text { pausas, expansión, sandwich } \\
\text { auditivo y condicionamiento } \\
\text { auditivo. }\end{array}$ & $\begin{array}{l}\text { Orientación espacial, campo } \\
\text { óptimo de la audición, } \\
\text { ampliación funcional del } \\
\text { campo óptimo de la audición, } \\
\text { control auditivo y seguir la } \\
\text { progresión lingüística } \\
\text { del niño oyente. }\end{array}$ & $\begin{array}{l}\text { Improvisación, Re-creativo, } \\
\text { composición y receptivo. }\end{array}$ \\
\hline
\end{tabular}

* No se incluyó la terapia auditiva-oral ya que dentro de la literatura encontrada, no existe claridad en su conceptualización. 
el manejo de la música por esta población, permiten vislumbrar el potencial que existe en el trabajo de los métodos musicoterapéuticos con esta población.

\section{CONCLUSIONES}

- Los métodos unisensoriales son usados por fonoaudiólogos capacitados y/o certificados en las diferentes áreas (Auditivo Verbal y Verbotonal) para la habilitación-rehabilitación de personas con deficiencias auditivas. Así mismo la musicoterapia, aunque es una disciplina relativamente joven, utiliza métodos propios para la rehabilitación de estas personas y propone nuevas visiones en la utilización de los sonidos (ver Tabla 1).

- Desde sus inicios, se ha mostrado la eficacia de la Terapia Auditiva Verbal como un método en el trabajo de rehabilitación de personas con deficiencias auditivas, se ha encontrado literatura reciente que apoya, en forma científica, la utilización de esta terapia como un método de intervención.

- La metodología verbotonal ha sido útil para el tratamiento de las personas con deficiencias auditivas, y aunque se encuentra bibliografía de su sustento teórico y desarrollo, se observa una acentuada escasez de publicaciones actuales que den cuenta de su aplicación en el pasado reciente.

- La terapia auditiva-oral es mencionada en algunas publicaciones, sin embargo su definición, principales exponentes y principios de trabajo parecen no están claros para los profesionales, ni en los reportes científicos en comparación con otros métodos.

- La musicoterapia es una herramienta de trabajo reciente en la población de usuarios con IC, sin embargo, puede aportar a la rehabilitación de personas con deficiencias auditivas desde la premisa del trabajo con sonidos musicales como su materia prima.

- Los fonoaudiólogos pueden tomar elementos musicales para el desarrollo de sus programas de intervención para alcanzar, de tal modo, una eficaz articulación entre música y fonoaudiología.

\section{BIBLIOGRAFÍA}

1. Rivas J, Valbuena M, Cabulla E, Ramírez $\mathrm{C}, \mathrm{y}$ cols. Dispositivos auditivos implantables: Implantes coclear de oído medio y de tallo cerebral. En: Tratado de otología y audiología: Diagnóstico y tratamiento médico quirúrgico. Colombia: AMOLCA. 2007.

2. CEAF-Comisión Española de AudioFonología Real Patronato sobre Discapacidad. Implantes Cocleares 2005; 7-8.N.REFLIST

3. Monfort M, Júarez A. Rehabilitación e intervención pedagógica. En: Implantes Cocleares. Barcelona: 2002. Masson.

4. JÚAREZ A. Intervención logopédica en la sordera infantil. En: Manual de logopedia. Barcelona: 1994. Masson. 187-208.

5. Crofton I, Fraser D. La música en citas. España: 2001.

6. DANE - Departamento Administrativo Nacional de Estadísticas. Información estadística de la discapacidad. Colombia: 2004.

7. INSOR - Instituto Nacional para Sordos. Estadísticas e información para contribuir en el mejoramiento de la calidad de vida de la población sorda colombiana. 2009. Boletín 1.

8. OMS - Organización Mundial de la Salud. Sordera y defectos de audición. 2010.

9. Flores L, Berruecos P. El niño sordo de edad escolar. México: 2001.

10. Ley 324 en Colombia. Diario Oficial No. 42.899, de 16 de octubre de 1996.

11. Manrique M, Huarte A. Implantes cocleares. Barcelona: 2002. Masson.

12. Torres S, Urouiza R, y col. Deficiencia auditiva: guía para profesionales y padres. 2000. Ed. Aljibe.

13. Bautista I. Sistemas de comunicación en alumnos/as sordos. Revista digital enfoques educativos 2010; 68: 14-24.

14. Puyuelo M, TORRes, S. y col. Intervención del lenguaje. Metodología y recursos educativos, Aplicaciones específicas a la deficiencia auditiva. Barcelona: 2001. Masson.

15. Fine P. La sordera en la primera y segunda infancia. Buenos aires: 1977. Editorial médica panamericana.

16. Reamy C, Brackett D. Communication methodologies: options for families. Otolaryngolic Clinics of North America 1999; 326: 1103-16. 
17. Estabrooks W, Schwartz R. The ABC's of AVT: analyzing auditory-verbal therapy. Canada: 1997. North York General Hospital.

18. MAGgIo dE MAgGI M. Terapia Auditivo Verbal: Enseñar a escuchar para aprender a hablar. Auditio: Revista electrónica de audiología 2004; 2: 64-73.

19. Dora Wu C, Brown PM. Parents Teachers Expectations of Auditory-Verbal Therapy. Volta Review 2004; 1041: 5-20.

20. LIM S, SIMSER, J y COL. Auditory-verbal therapy for children with hearing impairment. Annals of the Academy of Medicine 2005; 344: 307-12.

21. Dornan D, Hickson L, y CoL. Is Auditory-Verbal Therapy Effective for Children with Hearing Loss? Volta Review 2010; 1103: 361-87.

22. Dornan D, Hickson L, Y CoL. Longitudinal Study of Speech Perception, Speech, Language for Children with Hearing Loss in an Auditory-Verbal Therapy Program. Volta Review 2009: 109 2/3: 61-85.

23. Fairgray E, Purdy SC, y col. Effects of AuditoryVerbal Therapy for School-Aged Children with Hearing Loss: An Exploratory Study. Volta Review 2010; 1103: 407-33.

24. Yucel E, Derim D, y COL. The needs of hearing impaired children's parents who attend to auditory verbal therapy-counseling program. International Journal of Pediatric Otorhinolaryngology 2008; 72(7): 1097-111.

25. Bakhshaee M, Ghasemi M, y col. Speech development in children after cochlear implantation. European Archives of Otorhinolaryngology 2007; 264(11): 1263-6.

26. Chee G, Goldring J, y col. Benefits of cochlear implantation in early-deafened adults: the Toronto experience. Journal of Otolaryngology 2004; 33(1): 26-31.

27. EAsterbrooks $S$, O'Rourke $C$, y col. Child family factors associated with deaf children's success in auditory-verbal therapy. The American Journal of Otology 2000; 21(3): 341-4.

28. Allegro J, PAPSin, B, Y col. Acoustic analysis of voice in cochlear implant recipients with postmeningitic hearing loss. Cochlear Implants International 2010; 11(2): 100-16.

29. EASTERBROOKS S, O'ROURKE C. Gender differences in response to auditory-verbal intervention in children who are deaf or hard of hearing. American Annals of the Deaf 2001; 146(4): 309-19.
30. Eriks-Brophy A. Outcomes of Auditory-Verbal Therapy: A Review of the Evidence a Call for Action. Volta Review 2004; 1041: 21-35.

31. Triado C, Fernández M. La mediación semiótica en el sordo. Algunas reflexiones. Revista Logopédica, Fon, Audiol 1992; 124: 213-22

32. Gaulc K. Método Verbotonal. Habla y audición Método Verbotonal. Valencia, España: 1985.

33. INSOR - Instituto Nacional para Sordos. Requerimientos pedagógicos, administrativos y de servicios de apoyo para la integración escolar de educos sordos usuarios del castellano oral en instituciones educativas. 2007. Ministerio de Educación Nacional.

34. Gası K. A. Morant. Metodología Verbotonal. España: 2010.

35. Alcantud F, Soto F. Tecnologías de ayuda en personas con trastornos de comunicación. España: 2002. Nau libres.

36. Asp CW. The verbotonal method for management of young, hearing-impaired children. Ear Hearing 1985; 61: 39-42.

37. HSF- Hearing Speech Foundation focuses on research. Hudson Valley Business Journal2011; 2234: 19.

38. MILDNER V, _INDIJA B, Y COL. Speech perception of children with cochlear implants children with traditional hearing aids. Clinical Linguistics \& Phonetics 2006; 202/3: 219-29.

39. Santore F. The Verbotonal Aural Rehabilitation Program with a Hearing Impaired Adults: A Five Year Summary Report. Journal of the Academy of Rehabilitative Audiology 1978; 11(2): 33-44.

40. DiJonhnson $A$, Craig W, Y COL. An Investigation of the Verbotonal Method with Preschool Deaf Children: A Preliminary Interim Report, Pennsylvania State Dept. of Education, Harrisburg: 1971.

41. Tobey E, SHIN S, y CoL. Spoken word recognition in adolescent cochlear implant users during quiet multispeaker babble conditions. Otology Neurotology 2011; 32(3): 413-8.

42. Stewart I. After early identification-what follows? A study of some aspects of deaf education from an otolaryngological viewpoint. The Laryngoscope 1984; 946: 784-99.

43. Musselman C, Churchill A. Conversational control in mother-child dyads. Auditory-oral versus total communication. American Annals of the Deaf 1991; 136(1): 5-16. 
44. Santarelli R, De Filippi R, y col. Cochlear implantation outcome in prelingually deafened young adults. A speech perception study. Audiology Neurootology 2008; 13(4): 257-65.

45. Moog J, Geers A. Speech language acquisition in young children after cochlear implantation. Otolaryngologic Clinics of North America 1999; 32(6): 1127-41.

46. Hayes H, Geers A, y col. Receptive vocabulary development in deaf children with cochlear implants: achievement in an intensive auditory-oral educational setting. Ear Hearing 2009; 30(1): 128-35.

47. LERTSUKPRASERT K, KASEMKOSIN N, Y COL. Listening speaking ability of Thai deaf children in preschool aural rehabilitation program. Journal of Medical Association Thailand 2010; 93(4): 474-80.

48. WFMT. Definition of Music Therapy. http:// www.musictherapyworld.de, 2010.

49. Bruscia K. Musicoterapia métodos y prácticas. México: 2007. Pax México.

50. Marizza G, Rodríguez MC. Musicoterapia en Implante Coclear. Estado del Arte. (Tesis de grado para obtener el título de Musicoterapeuta). Universidad del Salvador. Buenos Aires: 2009.

51. RadBruCH K. Music therapy in the rehabilitation of children withcochlear implant $\mathrm{Cl}$. Music Therapy Today online 2001.

52. EsLAVA J. Musicoterapia en Implante Coclear. Conferencia Universidad del Salvador, Buenos Aires, Argentina: 2006.
53. Gfeller K, Mehr M, y COL. Aural Rehabilitation of music perception enjoyment of adult cochlear implant users. JARA 2001; 34: 17-27.

54. GfelleR K. Accomodating children who use cochlear implants in music therapy of educational settings. Music therapy perspectives 2000; 18(2): 122-30.

55. Vongpaisal T, TRehUB SE, y COL. Song recognition by children adolescents with cochlear implants. Journal of Speech Language Hearing Research 2006; 49(5): 1091-103.

56. Drennan W, Rubinstein R. Music perception in cochlear implant users its relationship with psychophysical capabilities. Journal of Rehabilitation Research Development 2008; 45(5): 779-89.

57. Lassaletta L, Castro A, y col. Does music perception have an impact on quality of life following cochlear implantation? Acta OtoLaryngologica 2007; 127(7): 682-6.

58. Trehub S, Vongpaisal T, y COL. Music in the Lives of Deaf Children with Cochlear Implants. The Neurosciences Music III-Disorders Plasticity. Ann NY Acad Sci 2009; 1169: 534-42.

59. QuiQue Y. Descripción de las características de un programa de tratamiento de musicoterapia en niños con implante coclear: Aproximación a la detección, discriminación, identificación y comprensión sonora. (Tesis de maestría) Universidad Nacional de Colombia 2012. 